Summary This study evaluated changes in the light output of rear signal lamps as a function of dirt accumulated during a $482 \mathrm{~km}$ drive, representing ten days' driving for a typical United States driver. The complete route was traversed on three separate occasions, under each of the following environmental condirions: dry, wet, and snowy/salty. Luminous intensity measurements were obtained for all US and European test points. Photometry for each of two stop lamps was performed twice after the completion of each drive: first 'as is' and then after cleaning. The resuits indicate that dirt deposits tended to cause the light output to decrease at the points tested. The reductions after the dry drive were all less than $8 \%$. However, after the wet and snowy/salty drives reductions of more than $25 \%$ occurred at several test points, with a maximum reduction of $37 \%$. The greatest percentage reductions occurred for the points at and near the optical axes of the lamps, which had the highest original intensities, and at which maintaining adequate intensity is presumably most important. A theoretical analysis of the changes caused by dirt indicates that this is the pattern of results that will usually occur. A full evaluation of the significance of the effects of dirt that are quantified in this paper should be done in the context of other factors that affect signal-lamp intensity, such as vehicle voltage control and lamp design. It may also be important to measure more fully the range and distributions of dirt conditions in the real world. However, the present results demonstrate that, within the range of common weather conditions, dirt can cause reductions of signal-lamp intensity that are large enough to be of concern, especially for the relatively important positions at and near the optical axes of signal lamps.

\title{
Automobile rear signal lamps: Effects of realistic levels of dirt on light output
}

\author{
M Sivak PhD, M J Flannagan PhD, E C Traube and S Kojima \\ The University of Michigan Transportation Research Institute, 2901 Baxter Road, Ann Arbor, Michigan 48109-2150, USA
}

Received 15 July 1997, in final form 30 October 1997

\section{Introduction}

Dirt deposits on lenses of vehicle lamps have two major effects: a reduction in the total amount of transmitted light, and an increase in scattered light. For headlamps, the net effect of these two processes is that, for those parts of the beam pattern where most of the light is directed (generally below horizontal), there is a reduction in light, whereas for those parts where, by design, light is kept to a minimum (generally above horizontal), there is an increase in light ${ }^{11}$. In contrast, rear signal lamps are not designed to minimise light output in any particular direction. Consequently, it is not clear whether dirt on rear signal lamps will lead to a mixture of increases and decreases in light output, or only to decreases. We are not aware of any published data on the effects of dirt on light output of rear signal lamps.

This study evaluated changes in the light output of stop lamps as a function of dirt accumulated during a $482-\mathrm{km}$ route, representing ten days' driving for a typical US driver. The complete route was traversed on three separate occasions, under each of the following conditions: dry, wet, and snowy with road salt. Photometry for each of two stop lamps was performed twice after the completion of each drive, first 'as is' and then after cleaning. Photometric information was obtained for all current US and European test points.

Although only stop lamps were measured, it is reasonable to assume that the results would be applicable to all standard (low-mounted) rear signal lamps, including those that signal presence/tail, turn, and backup. On the other hand, the results might not be applicable to high-mounted stop lamps, because their higher mounting (and frequent location behind the rear window) might result in less accumulation of dirt.

\section{Method}

\subsection{Test vehicle and lamps}

A mid-size car was used in this study. The car was equipped with its original rear lamps. On each side of the vehicle there were two laterally adjacent stop/tail lamps, a turn signal lamp, and a backup lamp.

The photometry was performed on the outboard-mounted stop/tail lamp on each side. The lamps had a red outer lens and a clear inner lens with a replaceable two-filament bulb (No. 2057) and a parabolic reflector. During the photometry the stop filament was energised at $12.8 \mathrm{~V}$. The effective illuminated area (the lens size) was $165 \mathrm{~mm}$ wide by $85 \mathrm{~mm}$ high, with a $850 \mathrm{~mm}$ centre-to-ground distance. Centre-to-centre lateral separation between the two lamps was $1360 \mathrm{~mm}$.

\subsection{Test route}

The test route was the same as the one used in our previous study on the effects of dirt on the light distribution of low-beam headlamps ${ }^{(1)}$. The route was approximately $482 \mathrm{~km}$ long. It included roads in the southern and central portions of the lower peninsula of the state of Michigan. The surface of the route was asphalt $(67 \%)$, concrete $(30 \%)$, and unpaved $(3 \%)$. In terms of the road type, the route included rural twolane roads (53\%), limited-access multi-lane highways (39\%), and city streets $(8 \%)$.

\subsection{Test conditions}

The test route was driven three times, each time during daylight hours on a work day. The first drive took place in March 1997 on a snowy and cold day. Snow had fallen on the entire route within the previous 24 hours. During $37 \%$ of the route, active snow was falling. Most of the route was heavily saited. Approximately $73 \%$ of the route involved snowy or wet pave- 
ment (presumably with salt), 11\% was 'damp and salty,' and $16 \%$ was 'dry and salty.' The level of salt on the roads varied, but it was visible on the road surface for all dry sections of the route.

The second drive took place in April 1997 on a generally sunny and cool day. No precipitation occurred during the drive, and the pavement was dry throughout.

The third and final drive took place in May 1997 on a rainy and cool day. There was active precipitation on $86 \%$ of the route, with an additional $4 \%$ of the route having wet roads but no active precipitation.

\subsection{Test equipment}

The measurements were made in a photometry lab, using a goniometer. The distance from the lamp to the measuring screen was $30 \mathrm{~m}$.

\subsection{Procedure}

Changes in weather and road condition were recorded during the test drives. Because the temperature of the lens is likely to influence the nature of dirt deposits, the lamps were switched on and off using the following schedule repeated five times: $64 \mathrm{~km}$ on, $32 \mathrm{~km}$ off. The lamps were switched on so that the lenses would be at a relatively high temperature, characteristic of those times, during nighttime and low-visibility driving, when the headlamps (and thus also the rear lamps) are normally switched on.

The lamps were cleaned at the beginning of each drive. At the end of the test route, the lamps were removed from the vehicle. After they had been measured in the 'dirty' condition, they were cleaned and measured a second time.

Before photometry, the lamps were placed in stands built specifically for the style, shape, and model used in the study, and attached to the goniometer platform. Lamps were measured at all 19 US and European test points. Both measurements (dirty and clean) of one lamp were taken before the other lamp was measured.

\subsection{Evaluation of the effects of the changes in light output}

A decrease in the light output will result in a decrease in the effectiveness of rear signal lamps, especially under difficult environmental conditions such as bright sunlight ${ }^{(2-6)}$. The magnitude of the effect will depend on a variety of factors, such as the measure of interest (e.g. reaction time, proportion of missed signals, or conspicuity), ambient illumination, luminous intensities of the other rear lamps, etc. For example, a study by the Motor Industry Research Association(s) evaluated the effects of intensity of stop lamps on the detectability of stop signals. The stop lamps in the condition of interest were presented with presence lamps set at $7 \mathrm{~cd}$. During a daytime condition, a change in the luminous intensity of stop lamps from $66 \mathrm{~cd}$ to $32 \mathrm{~cd}$ (about a $50 \%$ reduction) resulted in an increase in reaction time from $1.04 \mathrm{~s}$ to $1.51 \mathrm{~s}$, and an increase in missed signals from $7.5 \%$ to $23.8 \%$. On the other hand, during a nighttime condition, the same change in luminous intensity produced no effect on either reaction time or missed signals.

To evaluate the practical importance of light output, a nonzero criterion has to be established. For this study we selected a change of $25 \%$ as such a criterion. This selection was based on a finding that subjects required signal lamp intensity to differ by $25 \%$ to be noticeably different ${ }^{(7)}$.

\section{Results and discussion}

\subsection{Luminous intensities with clean lenses}

The luminous intensities at the 19 test points after the lenses were cleaned (for each of the two lamps following each of the three test drives) ranged from $14.4 \mathrm{~cd}$ to $67.9 \mathrm{~cd}$. As indicated above, all measurements were made for the outboard of the two stop lamps on each side of the vehicle. Assuming that the inboard stop lamps (which were not measured) would provide the same output as the outboard lamps, the cleaned stop lamps on the test vehicle (after each drive) met the US regulations $^{(8)}$ for two-comparment stop lamps.

\subsection{Comparison of buminous intensities with clean and dirty lenses}

The means and ranges of the differences in luminous intensities after each of the three drives are shown in Table 1 for each lamp. The actual differences at each test point are listed in Table 2. As is evident from the information in Tables 1 and 2 , the differences after the dry drive were negligible. On the other hand, the differences after the wet and snowy/salty drives were substantial, exceeding $25 \%$ at several of the test points, with a maximum reduction of $37 \%$. All of the differences after the latter two drives were reductions in output.

The reductions in the light output were greater for the left lamp than for the right lamp both after the wet drive (mean reductions of $26 \%$ versus $17 \%$ ) and after the snowy/salty drive $(30 \%$ versus $26 \%$ ). However, the partern was reversed after the dry drive $(0 \%$ versus $5 \%)$. One speculative explanation for these results is as follows. (The listed directions of the effects apply to right-hand traffic; they would be reversed for lefthand traffic.) When the roadway is wet (because of either rain or snow), spray from overtaking traffic (and to a lesser degree from oncoming traffic) results in more dirt deposits on left lamps than on right lamps. On the other hand, when the roadway is dry, there is probably more dirt on the right side of the lane of travel on a two-lane roadway (due to the crown of the road).

\section{3 'Clean' luminous intensity as a predictor of 'dirty' luminous intensity}

For each test drive and each lamp we regressed 'dirty' luminous intensities on corresponding 'clean' luminous intensities ${ }^{(1)}$. The relationships berween intensities of clean lamps and dirty lamps were well described by linear models (all $r^{2}$ values were greater than 0.98 ). The fact that linear models provide a good fit implies that the effects of dirt can be separated into two components that can be quantified by two parameters: a proportional reduction in the luminous intensity throughout the beam pattern (quantified by a slope) and additional light superimposed uniformly throughout the beam pattern (quantified by an intercept).

An example of a scatter plot of 'dirty' versus 'clean' luminous intensity for one lamp (left) and one environmental condition (snowy/salty) is shown in Figure 1, along with the best-fitting linear model. The slope of this equation ( 0.57 or $57 \%$ ) is an estimate of the proportional reduction in luminous intensity throughout the beam pattern, presumably caused by both absorption and scattering. The intercept of this equation (4.0) is an estimate of the amount of a superimposed intensity (in cd) throughout the beam pattern, presumably caused by scattering. In other words, the regression equation suggests that after the snowy/salty drive for the left lamp the dirt deposits reduced luminous intensity at each test point to $57 \%$ of the original value, and that this reduction was partially offset by a 
Table 1 Means and ranges of the percentage differences in luminous intensities between 'clean' and 'dirty' measurements after each drive. The calculations involved subtracting the (generally higher) 'clean' luminous intensities from the corresponding (generally lower) 'dirty' luminous intensities.

\begin{tabular}{llllll}
\hline Drive & \multicolumn{2}{c}{ Left lamp } & & \multicolumn{2}{c}{ Right lamp } \\
\cline { 2 - 3 } \cline { 5 - 6 } & Mean & Range & & Mean & Range \\
\hline Dry & 0 & $-1,+1$ & & -5 & $-7,-3$ \\
Wet & -26 & $-33,-14$ & & -17 & $-22,-10$ \\
Snowy/salty & -30 & $-37,-14$ & -26 & $-35,-13$ \\
\hline
\end{tabular}

superimposed uniform addition of $4.0 \mathrm{~cd}$ throughout the beam pattern.

To the extent that linear models provide good descriptions of the relationships between 'clean' and 'dirty' luminous intensities, we can estimate which levels of intensity will increase because of dirt and which will decrease. Using the best-fitting linear equations, we calculated the pivot intensities. Luminous intensities of clean lamps that are smaller than the corresponding pivot intensity would be expected to increase due to dirt, because at these intensity levels the uniform intensity increase is greater than the proportional decrease. On the other hand, the luminous intensities that are greater than the pivot intensity would be expected to decrease, because at these intensity levels the uniform intensity increase is smaller than the proportional decrease. (Points with luminous intensities equal to pivot intensity should remain unchanged.) The specific calculation involved solving the regression equation $(y=a x+b)$ for $y=x$. The pivot intensity for the example shown in Figure 1 (the left lamp after the snowy/salty drive) proved to be $9.4 \mathrm{~cd}$.

Table 3 lists the parameters of the best-fitting linear equations and the corresponding pivot intensities for the two environmental conditions that showed substantial effects of dirt (i.e. the wet and snowy/salty drives).

One potencially interesting aspect of the existence of pivot points (evident graphically in Figure 1), is that, in terms of a percentage reduction, dirt will have the most effect on the brightest test points and the least effect on the dimmest test points. Using the example in Figure 1 (the left lamp after the snowy/salty drive), a point that is $60 \mathrm{~cd}$ when clean is predicted to lose $36 \%$, while a point that is $20 \mathrm{~cd}$ when clean is predicted to lose $23 \%$.

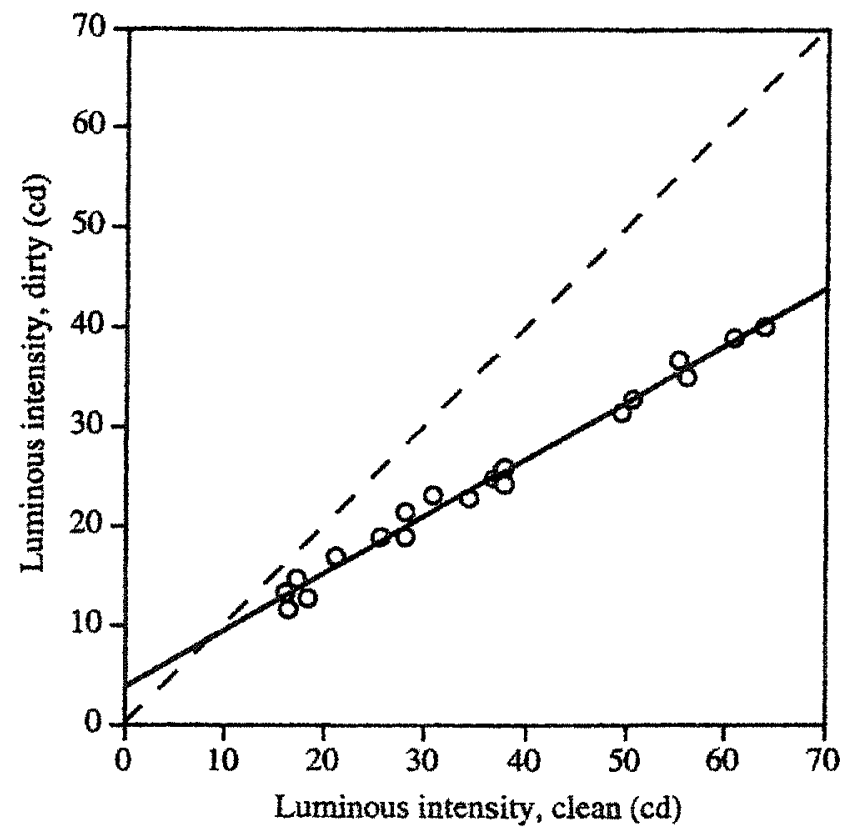

Figure 1 The relationship between the 'clean' and 'dirty' luminous intensities for the left lamp after the snowy/salty drive. The solid line is the best-fitting linear model $(y=0.57 x+4.0)$. For comparison, the dashed line shows where points would fall if luminous intensities were unaffected by $\operatorname{dirt}(y=x)$.

Table 3 The parameters of the best-fitting linear equations and the corresponding pivot intensities for the wet and snowy/salty drives (All regressions were statistically significant; $p<0.001$.)

\begin{tabular}{|c|c|c|c|c|}
\hline Drive & Lamp & Slope & Intercept $(c d)$ & Pivot intensity (cd) \\
\hline Wet & Left & 0.62 & 3.2 & 8.6 \\
\hline Wer & Right & 0.76 & 1.9 & 7.7 \\
\hline Snowy/salty & Left & 0.57 & 4.0 & 9.4 \\
\hline Snowy/salty & Right & 0.60 & 4.1 & 10.2 \\
\hline
\end{tabular}

\subsection{Comparison of the effects of dirt on rear signal lamps and head- lamps}

Both the present study and our recent study on the effect of dirt on the light output of low-beam headlamps ${ }^{(1)}$ used the same route. Although the environmental conditions in the two studies were also the same nominally (dry, wet, and snowy/salty), there were some differences. The dry drive in

Table 2 Percentage differences in luminous intensities between 'clean' and 'dirty' at each test point. At each location, the first value is for the left lamp, and the second value is for the right lamp.

\begin{tabular}{|c|c|c|c|c|c|c|c|}
\hline & $20^{\circ} \mathrm{Left}$ & $10^{\circ}$ Left & $5^{\circ}$ Left & Vertical & $5^{\circ}$ Right & $10^{\circ}$ Right & $20^{\circ}$ Right \\
\hline $\begin{array}{l}\text { Doy } \\
10^{\circ} \mathrm{Up} \\
5^{\circ} \mathrm{Up} \\
\text { Hoxizontal } \\
5^{\circ} \text { Down } \\
10^{\circ} \text { Down }\end{array}$ & $\begin{array}{r}+1 /-7 \\
0<-6\end{array}$ & $\begin{array}{l}0 /-7 \\
0 /-6 \\
0 /-6\end{array}$ & $\begin{array}{c}+1 /-4 \\
0 /-4 \\
0 /-3\end{array}$ & $\begin{array}{r}+1 /-7 \\
0 /-6 \\
-1 /-5\end{array}$ & $\begin{array}{c}+1 /-5 \\
0 /-5 \\
0 /-4\end{array}$ & $\begin{array}{l}0 /-6 \\
0 /-5 \\
0 /-5\end{array}$ & $\begin{array}{l}0 /-4 \\
-1 /-4\end{array}$ \\
\hline $\begin{array}{l}\text { Wet } \\
10^{\circ} \text { Up } \\
5^{\circ} U_{p} \\
\text { Honzontal } \\
5^{\circ} \text { Down } \\
10^{\circ} \text { Down }\end{array}$ & $\begin{array}{l}-24 /-13 \\
-24 /-14\end{array}$ & $\begin{array}{l}-28 /-19 \\
-30 /-22 \\
-26 /-21\end{array}$ & $\begin{array}{l}-20 /-13 \\
-31 /-19 \\
-15 /-10\end{array}$ & $\begin{array}{l}-30 /-19 \\
-33 /-21 \\
-29 /-22\end{array}$ & $\begin{array}{l}-23 /-11 \\
-23 /-20 \\
-14 /-11\end{array}$ & $\begin{array}{l}-31 /-18 \\
-33 /-21 \\
-27 /-20\end{array}$ & $\begin{array}{l}-24 /-14 \\
-21 /-17\end{array}$ \\
\hline $\begin{array}{l}\text { Showylsally } \\
10^{\circ} \text { Up } \\
5^{\circ} \text { Up } \\
\text { Horizontal } \\
5^{\circ} \text { Down } \\
10^{\circ} \text { Down }\end{array}$ & $\begin{array}{l}-30 /-23 \\
-28 /-21\end{array}$ & $\begin{array}{l}-33 /-32 \\
-36 /-34 \\
-32 /-28\end{array}$ & $\begin{array}{l}-25 /-18 \\
-36 /-34 \\
-16 /-13 \\
\end{array}$ & $\begin{array}{l}-35 /-32 \\
-37 /-35 \\
-33 /-31\end{array}$ & $\begin{array}{l}-25 /-17 \\
-37 /-34 \\
-14 /-15\end{array}$ & $\begin{array}{l}-34 /-29 \\
-36 /-32 \\
-32 /-29\end{array}$ & $\begin{array}{l}-24 /-22 \\
-19 /-23\end{array}$ \\
\hline
\end{tabular}


the present study was done in the spring, whereas the dry drive in the headlighting study was done during the summer, with more insects present in the air. (Although, even if the numbers of insects had been equal for the two studies, they presumably would have had less effect on rear lamps than on front lamps.) Furthermore, the wet drive in the present study included a higher proportion of active rain and wet roadways. Finally, the snowy/salty drive in the present study was partly during active snowfall, whereas the snowy/salty drive in the headlighting study was performed a day after a snowfall. Although it is important to keep the environmental differences in mind, it is nevertheless instructive to compare the results of the two studies.

For rear signal lamps, the only practically significant changes in light output were decreases. This is probably because the distribution of light from the signal lamps was more uniform than the distribution of light from the headlamps. As a consequence of the more uniform distribution, all of the measured 'clean' luminous intensities for the signal lamps were greater than the corresponding pivot luminous intensities (for example, see Figure 1). In contrast, the effect of dirt on headlamps resulted in both decreases and increases, with increases generally confined to light above and near the horizontal.

After the dry drives, the decreases were greater for the headlamps than for the rear lamps. This is probably a consequence of the fact that the presence of insects in the air has a greater effect on the cleanliness of the headlamps than on the cleanliness of the rear lamps. (There were also more insects present during the beadlighting drive.) After the wet drives, the decreases in the output of rear lamps were somewhat greater than those of the headlamps. Finally, after the snowy/salty drives, the magnirudes of the peak decreases were comparable.

\section{Conclusions}

The results indicate that dirt deposits tended to cause the light output to decrease at the points tested. The reductions after the dry drive were all less than $8 \%$. However, after the wet and snowy/salty drives reductions of more than $25 \%$ occurred at several test points, with a maximum reduction of $37 \%$. The greatest percentage reductions occurred for the points at and near the optical axes of the lamps, which had the highest original intensities, and at which maintaining adequate intensity is presumably most important. A theoretical analysis of the changes caused by dirt indicates that this is the pattern of results that will usually occur.

A full evaluation of the significance of the effects of dirt that are quantified in this paper should be done in the context of other factors that affect signal-lamp intensity, such as vehicle voltage control and lamp design. It may also be important to measure more fully the range and distributions of dirt conditions in the real world. However, the present results demonstrate that, within the range of common weather conditions, dirt can cause reductions of signal-lamp intensity that are large enough to be of concern, especially for the relatively important positions at and near the optical axes of signal lamps.

\section{Acknowledgements}

Appreciation is extended to the members of the University of Michigan Industry Affiliation Program for Human Factors in Transportation Safety for support of this research. The cur- rent members of the Program are: Adac Plastics, Bosch, Britax International, Chrysler, Corning, Delphi Interior and Lighting Systems, Denso, GE, GM NAO Safety Center, Hella, Hewlett-Packard, Ichikoh Industries, Koito Manufacturing, LESCOA, Libbey-Owens-Ford, Magneti Marelli, North American Lighting, Osram Sylvania, Philips Lighting, PPG Industries, Reflexite, Stanley Electric, Stimsonite, TEXTRON Automotive, Valeo, Visteon, Wagner Lighting, 3M Personal Safety Products, and 3M Traffic Control Materials. We thank II Stanley for allowing us to use their facilities to perform the photometry.

\section{References}

1 Sivak M, Flannagan, M J, Traube E C, Kojima $S$ and Aoki M Low-beam headlamps: Effects of realistic levels of dirt on light ouput Lighting Res. Technot. 29(1) 15-22 (1997)

2 Mortimer R G, Moore $\mathrm{CW}$, Jorgeson $\mathrm{C} M$ and Thomas J $\mathrm{K}$ Passenger car and truck signaling and marking research. I. Regulations, intensity requirements and color filter chandcteristics Report HSRI-HF-73-18 (Ann Arbor, MI: The University of Michigan, Highway Safety Research Institute) (1973)

3 Schmidt-Clausen $\mathrm{HJ}$ Optimum luminances and areas of rear-position lamps and stop lamps Proc. 10th Int. Conf. Experimental Safety Vehicles pp 220-224 (Washington, DC: National Highway Traffic Safety Administration) (1986)

4 Sivak M, Flannagan M J, Olson P L, Bender $M$ and Conn L S Exabuation of brake-lamp photometric requirements Report UMTRI-86-28 (Ann Arbor, MI: The University of Michigan Transportation Research Institute) (1986)

5 Motor Industry Research Association A study of the effectiveness of rear lighting arrangements for cars Contractor Report 92 (Crowthorne, England: Transport and Road Research Laboratory) (1988)

6 Sayer J R, Flannagan M J and Sivak M Effects of intensity, area, and aspect ratio on reaction time to stop lamps Report UMTRI-95-10 (Ann Arbor, MI: The University of Michigan Transportation Research Institute) (1995)

7 Huey $\mathrm{R}$, Dekker $\mathrm{D}$ and Lyons $\mathrm{R}$ Driver perception of just-noticeable differences of automotive signal lamp intensities Report DOT HS 808209 (Washington, DC: National Highway Traffic Safety Administration) (1994)

8 Office of the Federal Register FMVSS (Federal Motor Vehicle Safety Sicandard) 108 (Lamps, reflective devices, and associated equipment) 49 Code of federal regulations Part 571.108 (Washington, DC: US Government Printing Office) (1996)

\section{Discussion}

\section{J Cobb (VisualEyes)}

We must be grateful for UMTRI for continuing to provide data on the performance of vehicle lighting in real conditions. It is essential that the differences between manufacturers test conditions and real life be put together with driver performance studies in order to improve regulations.

As with the previous measurements of headlights, I wonder how the route of $483 \mathrm{~km}$ (equivalent to ten days' driving for a US driver) is believed to be 'realistic' as the title states. Do US drivers wash their cars every 20 days?

There is some previous evidence of changes in rear light intensity due to dirt ${ }^{93}$, which showed reductions of 30 to $50 \%$ for all vehicle and light types. The $25 \%$ difference, used in this paper for the definition of 'no change', is based on subjective experience of change, but it may be that objective changes (such as missed signals) take place at some other level 
of difference. However, the differences found between dry and wer or snowy conditions do not depend critically on this definition.

Again, as with the headlight study, presumably the lights were measured dirty but dry in the laboratory, so perhaps not quite representing the on-the-road light output while in the wet or snowy weather.

It is interesting that the cleaned lamps would have met US regulations, as for stoplights in the UK (in 1989) between $46 \%$ (cars) and $88 \%$ (articulated trailers) would have failed type approval tests $($ ).

The graph in Figure 1 shows a very good linear fit. Presumably if the pivot intensities were interpreted as a proportion of the 'average' intensity, this may allow the model to be used for other light types, such as direction indicators.

The decreases for the rear lights are found to be more than those for headlights, but the dirt on rear lights may be more subject to the aerodynamic properties of the vehicle. This effect will show up most as a difference between vehicle types, which should therefore be included in any comprehensive study of intensity changes due to conditions of use.

\section{Reference}

9 Cobb J Roadside Survey of Vehicle Lighting 1989 Research Report 290 (Crowthorne, UK: Transport and Road Research Laboratory) (1989)

\section{Authors' response to discussion}

We appreciate the discussant's kind words and thoughtful questions with regard to our paper. In particular, his raising of the validity issue with regard to the $25 \%$ criterion is important. We agree that not all driver performance measures will necessarily follow the results of Huey et al. ${ }^{(n)}$. However, we believe that their measure of just noticeable differences provides a useful, though not fully conclusive, guide to what differences in signal lamp intensity are likely to have significant consequences.

As we indicated in the companion headlighting paper ${ }^{(n)}$, we are not aware of any data on the average interval between car washes in the United States. The phrase 'realistic levels' is meant to refer to realistic levels after ten days' driving without washing.

In the wet condition, the lamps were in fact allowed to dry before they were tested.

As pointed out in section 3.4, the decreases for the rear lamps were found to be greater than those for headlamps only for the wet drives. After the dry drives, the decreases were greater for headlamps, while after the snowy/salty drives the magnitudes were comparable. 